NGR.09.005.063

N $73 \quad 22665$

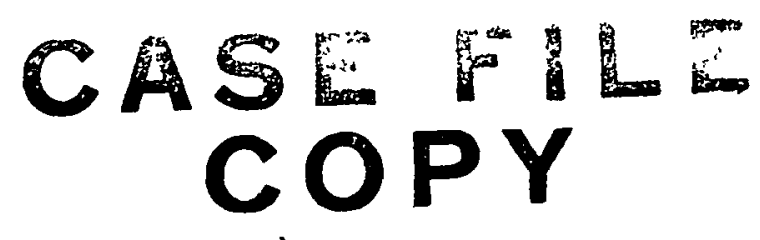

ALFVEN WAVES IN SPIRAL

INTERPLANETARY FIELD

Y.C. Whang

March 1973

Department of Plasma Physics Royal Institute of Technology 10044 Stockholm 70, Sweden

(On Sabbatical Leave from the Catholic University of America, Washington, D.C.20017 USA)

Short Title: AlIven Waves 


\section{ALFVEN WAVES IN SPIRAL INTERPLANETARY FIELD}

Y.C. Whang

\section{Abstract}

This paper presents a theoretical study of the Alfven waves in the spiral interplanetary magnetic field. The Alfvén waves under consideration are arbitrary, large amplitude, non-monochromatic, microscale waves of any polarization. They superpose on a mesoscale background flow of thermally anisotropic plasma. Using WKB approximation, an analytical solution for the amplitude vectors is obtained as a function of the background flow properties: density, velocity, Alfvén speed, thermal anisotropy, and the spiral angle. The necessary condition for the validity of the WKB solution is discussed. The intensity of fluctuations is calculated as a function of heliocentric distance. The relative intensity of fluctuations compared with the magnitude of the background field has its maximum in the region near $1 \mathrm{a} . \mathrm{u}$. Thus outside of this region, the solar wind is less turbulent. Due to attenuation of microscale Alfvén waves, fluctuation energy is converted into the kinetic energy of the solar wind. Its contribution amounts to about 20 per cent of the kinetic energy at 1 a.u. 
Power spectra and discontinuities of the interplanetary magnetic field have been studied by Burlaga (1968, 1969), Coleman (1966), Sari and Ness (1969), Siscoe et al. (1968), and others. These studies reveal that a considerable amount of microscale fluctuations is present in the interplanetary medium.

The frequency dependence of the power spectrum based on interplanetary magnetic field data were performed to study the statistical properties of the magnetic field fluctuations. Their results show that the mean amplitude of the fluctuations is usually a substantial fraction of the background field magnitude. The power densities of field fluctuations at a given frequency for the disturbed periods vary from 2 to 15 times those in the quiet periods. Except on very quiet days, the field fluctuations are not isotropic. The power levels in the field magnitude generally are substantially lower than those of the components, and the fluctuations are more intense in the direction transverse to the average background field.

Hydromagnetic discontinu:ties in the interplanetary medium are frequently observed. Most discontinuities are directional discontinuities (rotational or tangential discontinuities). Studying the spectral slope of the Pioneer 6 magnetic field data, Sari and Ness (1969) found that at times the power spectrum in the microscale range is dominated by the presence of directional discontinuities in the microstructure of the interplanetary magnetic field. One of the directional discontinuities, the rotational discontinuities are alsc known as Alfvén shocks or transverse shocks (Colburn and Sonett, 1966). Like smooth Alfuén waves, the rotatinnal discontinuitics propagate along the field with Alfven speed. Fluctuations in the velocity and field vectors for rotational discontinuities and for smoother Alfven waves are also governed by the same dynanical relations. Thus in a broader sense, Alfvén waves include rotational discontinuities. 
9. Recently Belcher et al. (1969), and Belcher and Davis (1971) studied the interplanetary fluctuations in velocity and magnetic field vectors using the simultaneous plasma and field data from Marinen 5. They showed that Alfvén waves with periods in the range of

$$
\tau_{1} \sim 10-10^{4} \text { seconds }
$$

propagate outward from the sun, and they are the major source of microscale fluctuations in the interplanetary medium.

This paper presents a theoretical study on the propagation of Alfvén waves in the interplanetary space. The Alfvén waves under study are of arbitrary form, large-amplitude and non-monochromatic waves, include smooth waves as well as rotational discontinuities, and are not restricted to any special polarizations. We consider that the microscale Alfuén waves with periods in the range of 10 to $10^{4}$ seconds superpose on a smooth background interplanetary field and plasma flow. In the time scale of $\sim 10$ seconds, rotational discontinuities actually appear like continuous current sheets. The background interplanetary magnetic field has a spiral configuration with its radial component inversely proportional to the square of heliocentric distance. The thermal anisotropy of the solar-wind plasma is included in the theory. We consider that the temporal and the spatial variations of the background field and plasma flow are measured by a mesoscale time of

$$
\tau_{2} \geqslant 10^{5} \text { seconds }
$$

or its equivalent length scale of

$$
L \geq 4 \times 10^{12} \mathrm{~cm}
$$

near 1 a.u. Thus we are dealing with a theoretical problem involving two time scales. The important concept of the various interplanetary time scales was first introduced by Burlaga and Hess (1968). (They divided the microscale and the mesoscale at $\sim 4 \times 10^{3}$ seconds instead of $\sim 10^{4}$ seconds.) 
We obtain analytical solution for the mesoscale attenuation of the Alfvern waves in the interplanetary space. It includes the effect of the spiral angle $\phi$. Some quantitative discussion of the result is also given.

\section{WKB APPROXIMATION}

O Let $\underline{V}$ and $\underline{B}$ respectively denote the momentary value of the'velocity and the magnetic field vector, and $\underline{U}$ and $\underline{B}_{0}$, the mesoscale average value of $\underline{V}$ and $\underline{B}$ with respect to time at a point fixed in space

$$
\underline{\mathrm{U}}=\langle\underline{\mathrm{V}}\rangle \text { and } \underline{\mathrm{B}}_{0}=\langle\underline{\mathrm{B}}\rangle
$$

Here the angle brackets are defined as

$$
\left\langle X(\underline{r}, t)=\frac{1}{T} \int_{-T / 2}^{T / 2} x(\underline{r}, t+\tau) d \tau\right.
$$

For practical reason, we take the averaging time $T$ to be a finite time interval. This interval must be at least several times the upper limit of the microscale period of the wave motion. Now we may write

$$
\underline{v}=\underline{U}+\underline{u} \text {. }
$$

and

$$
\underline{B}=\underline{B}_{0}+\underline{b}
$$

where $\underline{u}$ and $\underline{b}$ are microscale fluctuation vectors. The governing equations for the variation of $\underline{u}$ and $\underline{b}$ are

$$
\begin{aligned}
& \frac{\partial \underline{u}}{\partial t}+\underline{U} \cdot \nabla \underline{u}-\frac{\xi}{4 \pi \rho} \underline{B}_{0} \cdot \nabla \underline{b}=\frac{I}{4 \pi \rho}\left(\underline{B}_{0} \cdot \nabla \xi\right) \underline{b} \\
& \frac{\partial \underline{b}}{\partial t}+\underline{U} \cdot \nabla \underline{b}-\underline{B}_{0} \cdot \nabla \underline{u}=-(\nabla \cdot \underline{U}) \underline{b}
\end{aligned}
$$

Two kinds of terms are contained in the above two equations, the time-derivative of the fluctuation vectors and the derivatives of various quantities in the direction of the mescscale average vectors $\underline{U}$ and $\underline{B}_{\circ}$. The divergence of $\underline{U}$ represents the fractional change in mass density following the mesoscale motion of the flow

$$
\nabla \cdot \underline{U}=-\frac{1}{\rho}\left(\frac{\partial}{\partial t}+\underline{U} \cdot \nabla\right) \rho
$$


The effect of plasma thermal anisotropy is included in Equation (3) through the presence of a parameter $\xi$ which is defined. as

$$
\boldsymbol{\xi}=1-4 \pi\left(P_{\|}-P_{\perp}\right) / B^{2}=1-B\left(T_{\|}-T_{\perp}\right) / 2 T
$$

The parameters $\xi$ and $\beta$ which characterize the thermal properties of the magnetized plasma are calculated based on the time-average value of $B^{2}$, i.e.

$$
\left\langle B^{2}\right\rangle=B_{0}^{2}+\left\langle b^{2}\right\rangle
$$

When the two terms on the right hand side of Equation (3) and (4) are both zero, the equations describe the propagation of hydromagnetic waves along the convecting magnetic field lines with Alfvén speed

$$
A=(\xi / 4 \pi \rho)^{1 / 2} B_{0}
$$

and the two fluctuation vectors are related by

$$
\underline{u}=\mp(\xi / 4 \pi \rho)^{1 / 2} \underline{b} \text {. }
$$

In this case, Equations ( 3 ) and (4) are reduced to

$$
\left[\frac{\partial}{\partial t}+\left(\underline{u} \pm \underline{A}_{-}\right) \cdot \nabla\right](\underline{b}, \underline{u})=0
$$

where $\underline{e}_{1}=\underline{B}_{0} / B_{0} \cdot$ In (8) and (9) the upper sign refers to anti-parallel disturbances and the lower one to parallel disturbances. When an arbitrary disturbance is convected and propagated as Alfvén waves, $\underline{b}$ and $\underline{u}$ remain as two constant vectors. In other words, the amplitude of Alfvén waves remains unchanged in a region where $\xi$ and $\rho$ are uniform.

The characteristic length for the tarms on the left hand side of (3) and (4) is the wave length of a monochromatic Alfuen wave, $\lambda$. Let $L$ be the scale height for the variation of the background flow. Then the relative importance of the terms on the right hand side of (3) and (4) compared with those on the left hand side may be measured by a parameter

$$
\mu=\lambda / L
$$


$\mu$ is a small parameter $(\mu<1)$ when we consider that the

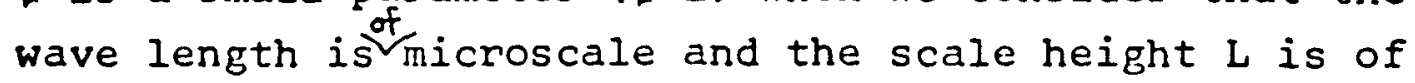
mesoscale. The small parameter $\mu$ is equivalent to $\tau_{1} / \tau_{2}$ when the background. flq, is also unsteady in mesoscale.

4

When $0<\mu<1$, the microscale Alfven waves are modified due to the slow variation of the background flow in the mesoscale. We may write each fluctuation vector for the solution of ( 3 ) and ( 4 ) as the product of a mesoscale amplitude vcctor and a microscale wave function $f$. Using WKB approximation the amplitude vectors are expanded as series in power of $\mu$ with the leading terms of the series satisfying the zeroth order solution ( 8 ). The microscale wave function $f$ represents an arbitrary function of its argument satisfying the equation for traveling Alfvén waves in a general way as though the mesoscale effect were absent,

$$
\left[\frac{\partial}{\partial t}+\left(\underline{U} \pm \mathrm{Ae}_{I}\right) \cdot \nabla\right] f=0
$$

The spatial and the temporal variation of $f$ are characterized by microscales, $\lambda$ and $\tau_{I}$. A special case for monochromatic sinusoidal waves,

$$
f=\exp (i S-i \omega t)
$$

with

$$
-\omega=\left(\underline{U} \pm A \underline{e}_{1}\right) \cdot \nabla S
$$

Under these approximations, we may write the two fluctuation vectors as

$$
\underline{b}=\left(\underline{b}_{1}+\mu \underline{b}_{2}+\ldots\right) f
$$

and

$$
\underline{u}=\left(\underline{u}_{1}+\mu \underline{u}_{2}+\ldots\right) f
$$

where

$$
\underline{u}_{1}=F(\xi / 4 \pi \rho)^{1 / 2} \underline{b}_{1}
$$

and $f$ remains as an arbitrary function.

The spatial and the temporal variation of $\underline{b}_{i}$ 's and $\underline{u}_{i}$ 's are characterized by mesoscales $L$ and ${ }^{2} 2$. 
91

Substituting (10)-(13) into (3), and keeping terms to first order in $\mu$, we obtain

$$
\begin{aligned}
& \mu A\left(\sqrt{\frac{4-\pi \rho}{\xi}} \underline{u}_{2} \pm \underline{b}_{2}\right) \underline{e}_{I} \cdot \nabla f+f\left(\frac{\partial}{\partial t}+\underline{U} \cdot \nabla \cdot \pm A \underline{e}_{1} \cdot \nabla\right) \underline{b}_{1}+ \\
& +f \underline{b}_{1}\left[\frac{1}{2 \xi}\left(\frac{\partial}{\partial t}+\underline{U} \cdot \nabla \pm 2 A \underline{e}_{1} \cdot \nabla\right) \xi-\frac{1}{2 \rho}\left(\frac{\partial}{\partial t}+\underline{U} \cdot \nabla\right)_{\rho}\right]=0
\end{aligned}
$$

Similarly, we obtain frum (4)

$$
\begin{aligned}
& -\mu A\left(\sqrt{\frac{4 \pi \rho}{\xi}} \underline{u}_{2} \pm \underline{b}_{2}\right) \underline{e}_{1} \cdot \nabla f+f\left(\frac{\partial}{\partial t}+\underline{u} \cdot \nabla \pm A_{e_{1}} \cdot \nabla\right) \underline{b}_{1}+ \\
& +f \underline{b}_{1}\left[ \pm \frac{A}{2 \xi} \underline{e}_{1} \cdot \nabla \xi-\frac{1}{\rho}\left(\frac{\partial}{\partial t}+\underline{u} \cdot \nabla \pm \frac{A}{2} \underline{e}_{1} \cdot \nabla\right) \rho\right]=0
\end{aligned}
$$

Add (14) to (15) and rearrange the terms, we obtain a singel differential equation for the variation of $\underline{b}_{1}$

$$
\begin{aligned}
& \xi^{1 / 2} \rho^{1 / 2}\left(\frac{\partial}{\partial t}+\underline{U} \cdot \nabla\right)\left(\xi^{1 / 4} \rho^{-3 / 4} \underline{b}_{1}\right) \pm A \underline{e}_{I} \cdot \nabla\left(\xi^{3 / 4} \rho-1 / 4 \underline{b}_{1}\right)= \\
& =0
\end{aligned}
$$

This equation holds in any coordinate system and with no restriction on the models of the background flow. No assumption has been made concerning the polarization of the fluctuation vectors.

9 In the region where the backgruund flow has a very high Alfvén number (U>>A), Equation (16) gives

$$
\xi^{1 / 4} \rho^{-3 / 4} \underline{b}_{1} \approx \text { constant }
$$

On the other limit, when the flow is steady and the Alfven speed is very large $(A>>U)$, the variation of wave amplitudes is governed by

$$
\xi^{3 / 4} p^{-1 / 4} \underline{b}_{1} \approx \text { constant }
$$




\section{PROPAGATION IN SPIRAL INTERPLANETARY FITLD}

If The solar-wind flow has a very low Alfven number in the corona. The Alfven number of the solar wind increases to approximate 10 near'the orbit of the earth, it continues to increase beyond $1 \mathrm{a} . \mathrm{u}$. he would like to find an exact solution of Equation (16) to cover the whole range of Alfven numbers for the variation of the amplitude vectors. of the Alfvern waves superposed on the solar wind.

丹 Consider a steady, spherically-symmetrical model of the solar wind, the background field has a spiral configuration. In general, the radial flow velocity $\underline{U}$ is not parallel to the direction of the spiral field. Let $\phi$ denote the angle between $\underline{U}$ and $\underline{e}_{I}$. Then we can write (16) in the following convenient form

$$
\begin{aligned}
& (U \pm A \cos \phi) \frac{d}{d r}\left(\xi^{3 / 4} \rho-1 / 4 \underline{b}_{1}\right)-\xi^{1 / 4} \rho^{-1 / 4} U \frac{d}{d r}\left(\xi^{1 / 2} \rho^{1 / 2}\right) \underline{b}_{1}= \\
& =0
\end{aligned}
$$

From the equation of divergence $\underline{B}$, the radial component of the background magnetic field, $B_{0} \cos \phi$, is proportional to $\mathrm{r}^{-2}$, we can write

$$
\frac{d}{d r}\left[(\rho / \xi)^{1 / 2} A r^{2} \cos \phi\right]=0
$$

The equation of continuity is

$$
\frac{d}{d r}\left(\rho U r^{2}\right)=0 \text {. }
$$

From (20) and (21) we obtain

$$
\xi^{-1 / 2} \rho^{-1 / 2} \frac{d}{d r}\left(\xi^{1 / 2} \rho^{1 / 2}\right)+\frac{A \cos \phi}{U} \frac{d}{d r}\left(\frac{U}{A \cos \phi}\right)=0
$$

Substituting (22) into (19), we obtæin

$$
\begin{aligned}
& \left(\frac{U}{A \cos \phi} \pm 1\right) \frac{d}{d r}\left(\xi^{3 / 4} \rho-1 / 4 \underline{b}_{1}\right)+\xi^{3 / 4} p-1 / 4 \frac{d}{d r}\left(\frac{U}{A \cos \phi}\right) \underline{b}_{1}= \\
& =0
\end{aligned}
$$


which can be integrated to give

$$
\xi^{3 / 4} \rho^{-1 / 4}\left(\frac{U}{A \cos \phi} \pm I\right) \underline{D}_{I}=\text { constant }
$$

Here the amplitude vector $\underline{b}_{1}$ is given as a function of $\xi, \rho$, $U, A$ and the spiral angle $\phi$. Since no limitation on the component of the background field transverse to the radial direction has been made in the above derivation, the result holds for very general spiral fields.

丹

Two special cases of Equation (23) have been studied in literatures. Farraro and Plumpton (1961) derived a solution for the verticai propagation of Alfvén waves in a horizontally stratified, stationary atmosphere. They assumed that the fluctuation vectors are linearly polarized in a horizontal direction and the medium is thermally isotropic.

Their result showed

$$
\rho^{-1 / 4} b_{t}=\text { constant }
$$

This is actually a special case of Equation (18), it must be valid for any form of background field.

Parker (1965), Belcher (1971), and Hollweg (1973b) studied the propagation of Aifvern waves in the solar-wind with the simplification that the sun does not rotate so that the background magnetic field is in radial direction everywhere. They also assumed the medium to be thermally isotropic and limited the fluctuation vectors to a linear polarization in the $\phi$-direction of a spherical polar-coordinate system. They obtained

$$
p^{-1 / 4}\left(\frac{U}{A} \pm 1\right) b_{\phi}=\text { constant. }
$$

Under the same assumptions, Hollweg (1973a) has also studied the higher-order terms, $\mathrm{b}_{2}, \mathrm{~b}_{3}, \mathrm{u}_{2}$ and $\mathrm{u}_{3}$, of the WKB approximation.

91 The accuracy of the WKB solutions can be gauged by comparing the magnitudes of the successive terms in the series repre- 
senting the amplitude vectors. If we substract (15) from (14), we obtain

$$
\begin{aligned}
& \mu \sqrt{\frac{4 \pi \rho}{\xi}} \underline{u}_{2} \underline{e}_{1} \cdot \nabla f \pm \mu \underline{b}_{2} \underline{e}_{1} \cdot \nabla f+\frac{f}{4 A} \underline{b}_{1} \frac{l}{\xi \rho}\left(\frac{\partial}{\partial t}+\underline{u} \cdot \nabla \pm A \underline{e}_{1} \cdot \nabla\right) \xi \rho= \\
& =0
\end{aligned}
$$

If $\underline{u}_{2}$ and $\underline{b}_{2}$ are parallel vectors, they must be parallel to $\underline{u}_{1}$ and $\underline{b}_{1}$. The relative magnitude of the three terms in (24) may be measured by

$$
\frac{\mu u_{2}}{u_{1}}: \frac{\mu b_{2}}{b_{1}}: \frac{1}{8 \pi}\left(\frac{U}{A \cos \phi}+1\right) \frac{\lambda}{L}
$$

The amplitude vectors may be approximated by their first terms, $\underline{b}_{1}$ and $\underline{u}_{1}$, in the domain where

$$
\mu b_{2} / b_{1}<1
$$

and

$$
\mu u_{2} / u_{1}<1
$$

These conditions are satisfied only when

$$
\frac{1}{8 \pi}\left(\frac{U}{A \cos \phi}+1\right) \frac{\lambda}{L}<1
$$

The relation between the two fluctuation vectors described by Equation ( 8 ) holds under this approximation. In the region outside $I$ a.u. the left hand side of (25) remains almost independent of the heliocentric distance $r$, because in that region $A \cos \phi$ is proportional to $r^{-1}$ and $L m / 2$.

\section{9} In general the microscale fluctuation vectors may be represented by series of monochromatic waves. The intensity of the microscale fluctuations may be defined as the root-mean-square value

$$
\bar{b}=\left(<b^{2}>\right)^{1 / 2}
$$


Now we use the WKB solution, Equation (23), to calculate the variation of $\bar{B}$ in the interplanetary space. A self-consistent solution of the solar-wind model including Alfvén waves and spiral fields is not available at the present time. In order to estimate the variations of $\xi$, $\rho$, $\mathrm{U}, \mathrm{A}$, and $\phi$ as functions of heliocentric distances, we choose the quantitative solution of the two-region model of the solar wind (Whang, 1972) in which the plasma thermal anisotropy and the spiral background field have been included. Figure 2 shows that $\bar{D}$ is calculated as a function of $r$ based on the assumption that

$$
\bar{b}=3 \text { gammas at } 1 \text { a.u. }
$$

$\bar{b}$ varies from 370 gammas at 0.01 a.u., 50 gammas at 0.1 a.u. to 0.1 gammas at $10 \mathrm{a} . \mathrm{u}$.

The relative intensity of $\bar{b}$ in comparison with the magnitude of the background field, $-B_{O}$, is a more important parameter in describing the local condition of the solar wind. From Figure 2 we can see that the relative maximum of $\bar{b} / B_{0}$ is located in the region near $1 \mathrm{a} . \mathrm{u}$. Thus outside of this region, for the Alfvén mode in concerned, the solar wind is expected to be less turbulent. The relative intensity $\bar{b} / \mathrm{B}_{0}$ varies asymptotically as $\mathrm{r}^{-1 / 2}$ at large hiliocentric distances.

\section{GOVERNING EQUATIONS}

\$

The equation of motion which governs dynamics of an anisotropic medium in the micro-, meso- and macro-scale is

$$
\frac{\partial}{\partial t}(\rho \underline{V})+\nabla \cdot\left(\rho \underline{V V}+P \underline{I}+\frac{B^{2}}{8 \pi} \underline{I}-\frac{\xi}{4 \pi} \underline{B} \underline{B}\right)-\rho g=0
$$

Making use of (1) and (2), we can write (27) as

$$
\begin{aligned}
& \rho\left[\frac{\partial \underline{u}}{\partial t}+\underline{U} \cdot \nabla \underline{u}-\frac{1}{4 \pi \rho} \underline{B}_{0} \cdot \nabla(\xi \underline{b})\right]+\left[\frac{\partial}{\partial t}(\rho \underline{U})+\nabla \cdot(\rho \underline{v} \underline{U}+\rho \underline{u} \underline{u}+\right. \\
& \left.+P \underline{\underline{I}}+\frac{B^{2}}{8 \pi} \underline{I}-\frac{\xi}{4 \pi} \underline{B} \underline{B}_{0}-\frac{\xi}{4 \pi} \underline{\underline{b}} \underline{)}-\rho \underline{g}\right]=0 \\
& ; \vdots
\end{aligned}
$$


No terms have been dropped out in Equation (28). The terms inside the first pair of square brackets is identical with Equation (3) which governs dynamics of microscale Alfvén waves. Now taking the time average of Equation. (28), we obtain

$$
\rho\left(\frac{\partial \underline{U}}{\partial t}+\underline{U} \cdot \underline{\nabla}\right)+\nabla \cdot\left(P_{\perp} \underline{I}+\frac{B_{O}^{2}}{8 \pi} \underline{I}-\frac{E}{4 \pi} \underline{B}_{0} \underline{B}_{0}+\underline{\underline{T}}\right)-\rho g=0
$$

where $\underline{\underline{T}}=\frac{I}{8 \pi}\left\langle b^{2}\right\rangle \underline{\underline{I}}+\rho\langle\underline{u} \underline{u}\rangle-\frac{\xi}{4 \pi}\langle\underline{b} \underline{b}\rangle$

All terms in this equation are actually contributed from the time average of the terms inside the second pair of square brackets in (28). It governs the meso- and macroscale motion of the background flow. In writing ( 3 ) as a governing equation for the microscale motion superposed on the background flow, no assumptions regarding the smallness of the fluctuation vectors have been introduced. The stress tensor $\stackrel{T}{\underline{T}}$ is the only coupling term between the microscale fluctuations and the background flow. To the zerothorder approximation, this fluctuation stress corresponds to an additional isotropicpressure due to microscale fluctuations (Alazraki and Couturier, 1971; Belcher, 1971)

$$
P_{A}=\frac{1}{8 \pi}\left\langle b^{2}\right\rangle \text {. }
$$

9. Faraday's law in the micro-, meso- and macroscale may be written as

$$
\frac{\partial \underline{B}}{\partial t}+\nabla \cdot(\underline{V} \underline{B}-\underline{B} \underline{V})=0
$$

or

$$
\begin{aligned}
& {\left[\frac{\partial \underline{b}}{\partial t}+\nabla \cdot\left(\underline{U} \underline{b}-\underline{B}_{0} \underline{u}\right)\right]+\left[\frac{\partial \underline{B}_{0}}{\partial t}+\nabla \cdot\left(\underline{V} \underline{B}_{0}-\underline{B} \underline{U}+\underline{u} \underline{b}-\right.\right.} \\
& -\underline{b} \underline{u})]=0
\end{aligned}
$$

The terms inside the first pair of square brackets is again identical with Equation (4) for the microscale Alfvén waves. The time average of the second part gives 


$$
\frac{\partial \underline{B}_{0}}{\partial t}+\nabla \cdot\left(\underline{U} \underline{B}_{0}-\underline{B}_{0} U\right)=\nabla \cdot(\langle\underline{b} \underline{u}\rangle-\langle\underline{u} \underline{b}\rangle)
$$

This equation governs the temporal and the spatial variations. of the background field in the meso- and macrn-scale. To the zeroth-order apprnximation, the right hand side of (31) vanishes when the two fluctuation vectors, $\underline{b}$ and $\underline{u}$, are either parallel or anti-parallel.

It is now clear that when we write (3) and (4) to describe the microscale Alfvern waves superposed on a background flow governed by (29) and (31), no limitations on the magnitude of the fluctuation vectors have becn imposed. The WKB solution obtain in this papcr, which satisfies (3) and (4) in a approximate way, must be valid for Alfvén waves with no limitation on the amplitude. This special feature of the Alfvén waves (Alfvén and Fälthammar, 1963) is preserved throughout this paper. .

To the zeroth-order approximation, the time-average energy equation, which governs the background flow in meso- or macro-scale, is

$$
\begin{aligned}
& \frac{\partial}{\partial t}\left(\frac{\rho}{2} U^{2}+P_{\perp}+\frac{P_{\|}}{2}+\frac{B_{O}^{2}}{8 \pi}+\varepsilon\right)+\nabla \cdot\left[\left(\frac{\rho}{2} U^{2}+2 P_{\perp}+\frac{P_{\|}}{2}+\frac{B_{O}^{2}}{4 \pi}\right) \underline{U}-\right. \\
& \left.-\frac{\xi}{4 \pi} \underline{B}_{0} \underline{B}_{0} \cdot \underline{U}+q+\underline{s}\right]-\rho g \cdot \underline{U}=0
\end{aligned}
$$

It contains two additional terms contributed by microscale fluctuations. They are the fluctuation energy density

$$
\varepsilon=\frac{\rho}{2}\left\langle u^{2}\right\rangle+\frac{I}{8 \pi}\left\langle b^{2}\right\rangle
$$

and the total energy flux due to fluctuations

$$
\underline{S}=\varepsilon \underline{U}+P_{A} \underline{U} \pm \hat{A} \rho<u^{2}>\underline{e}_{1}
$$

The total flux consists of three parts: convection of the fluctuation energy density, rate of work done by the fluctuation prassure, and propagation of wave energy along the moving background field lines. Recently Hollweg (1973a) 
theorized that very low-frequency Alfvern waves carry a larger wave energy flux than would be obtained in the zeroth-order WKB Iimit.

In the interplanetary space, the energy flow due to microscale Alfuén waves per steradian per unit time, $r^{2} s_{r}$, is a monotonically decreasing function of hiliocentric distance. Its value near the corona $(0.01 \mathrm{a} . \mathrm{u}$.$) is$ about $14 \%$ of the convective kinetic energy flow per steradian at 1 a.u. when we assume that the fluctuation intensity $\bar{b}=3$ gammas at 1 a.u. The estimated percentage of energy flow rises to $24 \%$ for $\bar{b}=4$ gammas at 1 a.u. This energy is converted into kinetic energy to increase the solar wind velocity. The interaction between the solar-wind flow and the attenuation of the fluctuation intensity is nonlinear in nature. A self-consistent model of the solar-wind must be studied to evaluate the effect of their interaction on a more accurate quantitative basis. 


\section{ACKNOULLDGMENTS}

I would like to thank Professor Fälthammar for the interest he has shown in this work. I appreciate the hospitality of the staff of the Royal Institute of Technology, Department of Plamsa Physics. This work was supported by the National Aeronautics and Space Administration under research grant NGR-09-005-063. 


\section{References}

Alfvén, H. and C.-G. Fälthammar, Cosmical Electrodynamics, Oxford University Press, London p. 89, 1963

Alazraki, G., and P. Couturier, Solar wind acceleration caused by the gradient of Alfvén wave pressure, Astron. and Astrophys., 13, 380, 1971

Belcher, J.W., Alfvénic wave pressures and the solar wind, Astrophys. J., 168, 509, 1971

Belcher, J.W., L. Davis, Jr., and E.J. Smith, Large-amplitude Alfvén waves in the interplanetary medium: Mariner 5, J. Geophys. Res., 24, 2302, 1969

Belcher, J.W. and L. Davis, Jr., Large-amplitude Alfvén waves in the interplanetary medium, 2, J. Geophys. Res., 76, 3534,1971

Burlaga, L.F., Microscale structures in the interplanetary medium, Solar Phys., 4, 67, 1968

Burlaga, L.F., Directional discontinuities in the interplanetary magnetic field, Solar Phys., I, 54, 1969

Burlaga, L.F. and N.F. Ness, Macro and micro structure of the interplanetary magnetic field, Canad. J. Phys., 46, S962, 1968

Coleman, P.J., Jr., Variations in the interplanetary magnetic field: Mariner 2, J. Geophys. Res., 71, 5509, 1966

Colburn, D.S. and C.P. Sonett, Discontinuities in the solar wind, Space Sci. Rev., $5,439,1966$

Ferraro, V.C.A. and C. Plumpton, Magneto-fluid Mechanics, Oxford Univ. Press, London, p. 69, 1961

Hollweg, J.V., Alfuén waves in the solar wind: wave pressure, poynting flux, and angular momentum, J. Geophys. Res., 78, $1973 \mathrm{a}$ 
- Hollweg, J.V., Alfvén waves in a two-fluid model of the solar wind, $1973 \mathrm{~b}$

Parker, E.N., Dynamical theory of the solar wind, Space Sci. Rev., 4, 667 , 1965

Sari, J.W., and N.F. Ness, Power spectra of the interplanetary magnetic field, Solar Phys., 8, 155, 1969

Siscoe, G.L., L. Davis, Jr., P.J. Coleman, Jr., E.J. Smith, and D.J. Jones, Power spectra and discontinuities of the interplanetary magnetic field: Marina 4, J. Geophys. Res., 73, 61, 1968

Whang, Y.C., A solar-wind model including proton thermal anisotropy, Astrophys. J., 178, 221, 1972 


\section{FIGURE CAPTIONS}

Figure 1 Propagation of Alfvén waves in a convecting spiral interplanetary magnetic field.

Figure 2 The intensity of field fluctuation due to microscale Alfvén waves is calculated as a function of heliocentric distance based on the assumed 3 gammas fluctuation at $1 \mathrm{a} . u$. The dashed curve is the magnitude of the background magnetic fieli. 


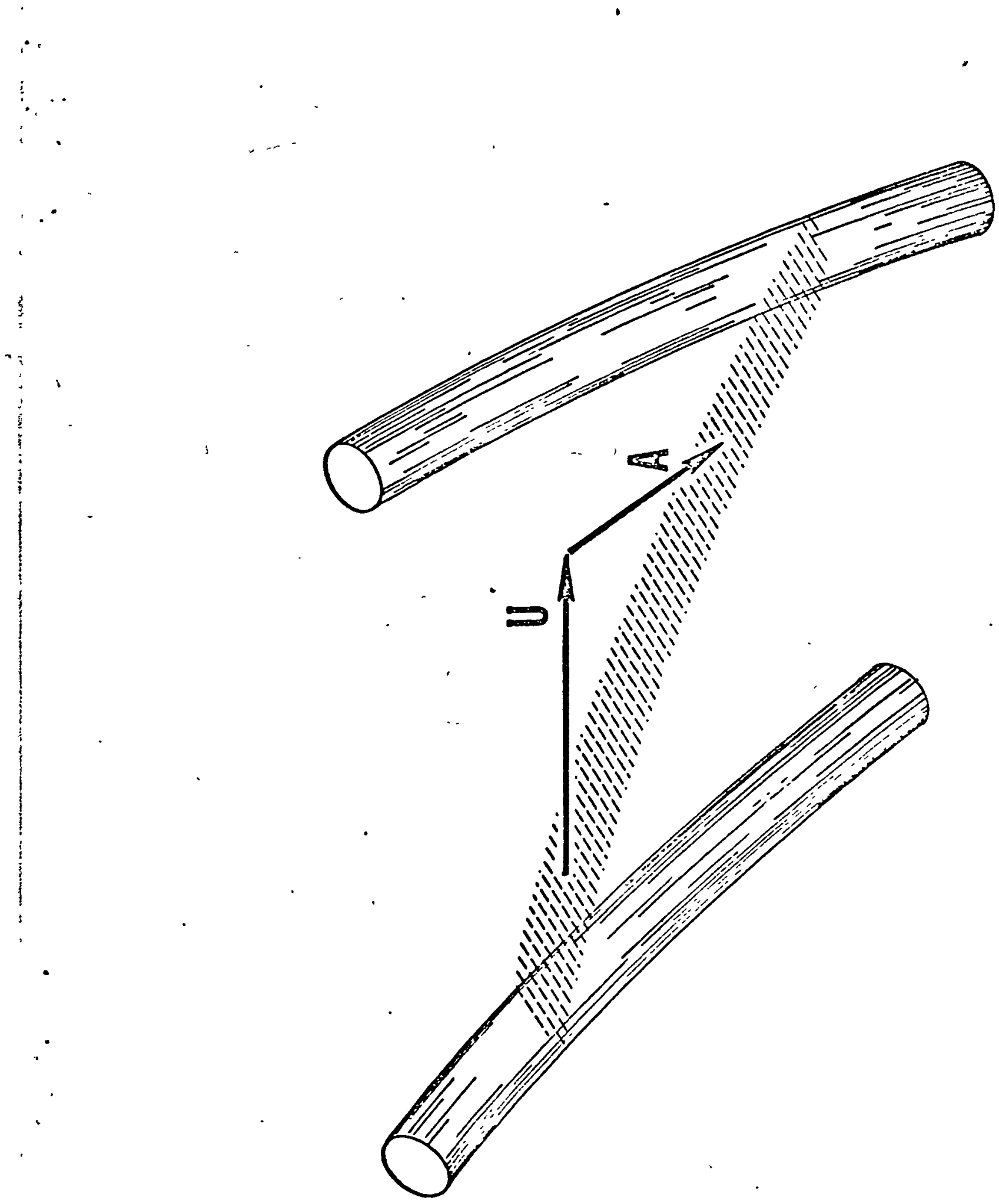




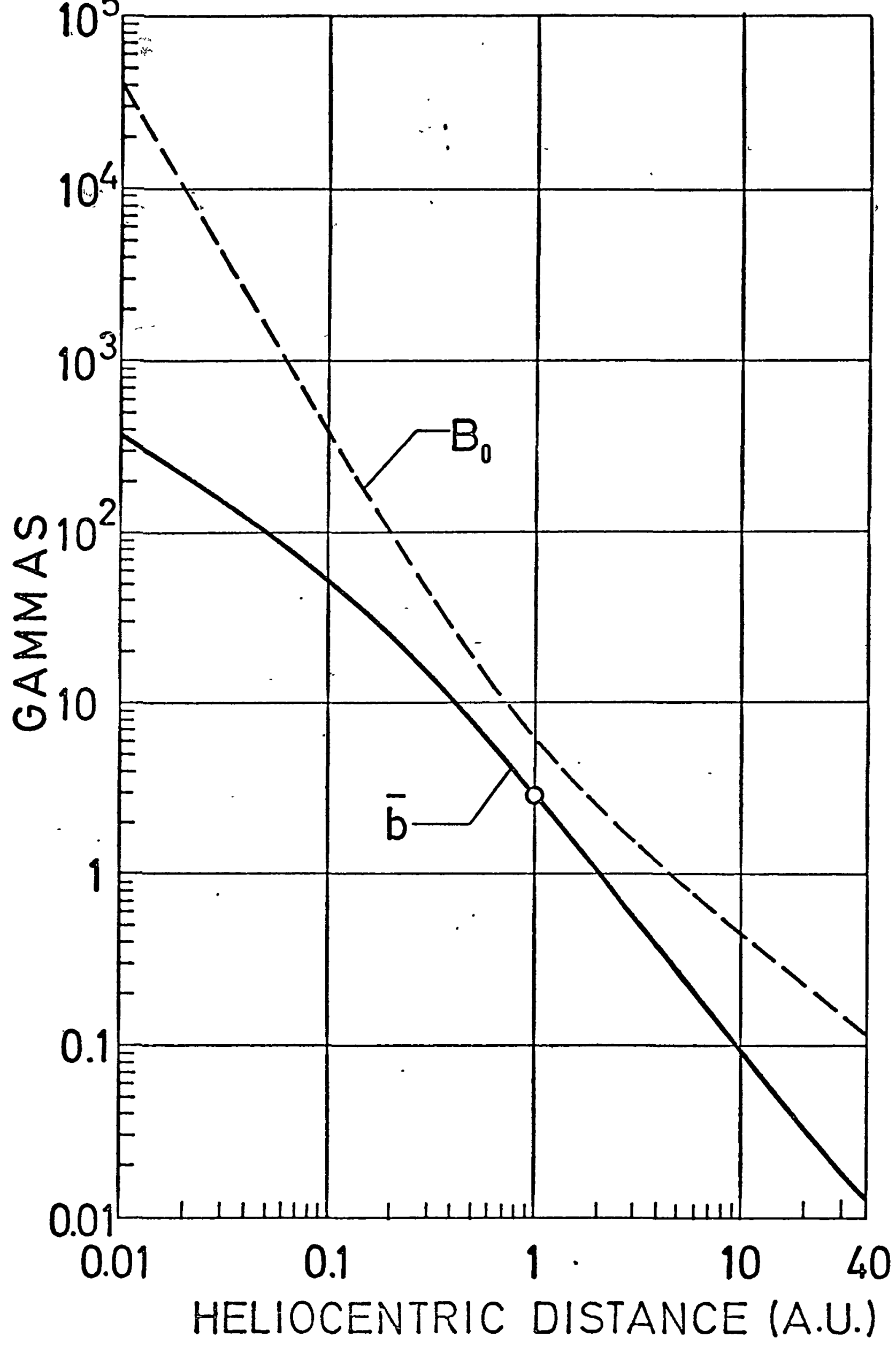

Fig. 2 